\title{
Anti-TNF therapies and pregnancy: outcome of 130 pregnancies in the British Society for Rheumatology Biologics Register
}

\author{
Suzanne M M Verstappen, Yvonne King, Kath D Watson, Deborah P M Symmons, \\ Kimme L Hyrich; BSRBR Control Centre Consortium, BSR Biologics Register
}

\begin{abstract}
- Additional data are published online only. To view these files please visit the journal online at (http://ard.bmj.com)

Arthritis Research UK Epidemiology Unit, The University of Manchester, Manchester Academic Health Science Centre, Manchester, UK
\end{abstract}

\section{Correspondence to} Dr Kimme Hyrich, Arthritis Research UK Epidemiology Unit, Manchester Academic Health Centre, University of Manchester, Stopford Building, Oxford Road, Manchester M13 9PT, UK;

Kimme.Hyrich@manchester. ac.uk

Accepted 15 November 2010 Published Online First 28 February 2011 online under the BMJ Journals unlocked scheme, see http:// ard.bmj.com/info/unlocked.dtl

\section{ABSTRACT}

Objective The British Society for Rheumatology Biologics Register (BSRBR) has collected data on adverse events including pregnancies in patients with rheumatoid arthritis treated with anti-tumour necrosis factor (antiTNF) therapy. The purpose of this report is to summarise the pregnancy outcomes in women treated with anti-TNF in the BSRBR.

Methods Patients were categorised according to anti-TNF exposure as follows: (1) exposure to anti-TNF and to methotrexate (MTX) and/or leflunomide (LEF) at conception ( $n=21$ pregnancies); (2) exposure to anti-TNF at conception ( $n=50)$; (3) exposure to anti-TNF prior to conception ( $\mathrm{n}=59$ ); (4) no exposure to anti-TNF (control group; $n=10)$.

Results Eighty-eight live births in a total of 130 pregnancies were reported in patients who received antiTNF before or during pregnancy. The rate of spontaneous abortion was highest among patients exposed to anti-TNF at the time of conception (with MTX/LEF 33\% and without MTX/LEF 24\%). This compared with 17\% spontaneous abortions in those with prior exposure to anti-TNF and $10 \%$ spontaneous abortions in the control group. Ten terminations were performed.

Conclusion Although the results to date have been promising, no firm conclusions can be drawn about the safety of anti-TNF during pregnancy and, without further evidence, guidelines which suggest these drugs should be avoided at the time of conception cannot yet be changed.

\section{INTRODUCTION}

Anti-tumour necrosis factor (anti-TNF) therapies have been available for the management of arthritis-related diseases for over a decade. The US FDA categorises anti-TNF agents as category 'B' drugs because animal reproduction studies have failed to demonstrate a risk to the fetus but adequate and well-controlled studies of pregnant women have not been conducted. ${ }^{1}$

To date, information on pregnancies in patients exposed to anti-TNF agents has been reassuring, with few reports of adverse pregnancy outcomes. One exception has been the report by Carter et $a l^{2}$ which listed 61 congenital anomalies reported to the FDA in 41 women exposed to anti-TNF agents including one child with the VACTERL syndrome (a syndrome seen in embryos and fetuses characterised by abnormalities of the vertebrae $(\mathrm{V})$, anus (A), cardiovascular tree (C), trachea ( $\mathrm{T})$, oesophagus (E), renal system (R) and limb buds (L)). However, this study lacked a denominator of exposure.
National registries such as the British Society for Rheumatology Biologics Register (BSRBR), which collects data on adverse events and pregnancy outcomes in patients treated with anti-TNF therapy, provide a more realistic representation of the effect of anti-TNF therapy on pregnancy outcome. Using data from the BSRBR, we previously reported on 32 pregnancies with known outcome in women exposed to anti-TNF agents. ${ }^{3}$ Since this publication, the number of pregnancies reported to the BSRBR has increased to 130 and the outcome of these pregnancies is reviewed in this paper.

\section{METHODS}

\section{Study design and patient population}

The patients for this study were participants registered in the BSRBR starting treatment with one of the three available anti-TNF therapies (adalimumab (ADA), etanercept (ETA) and infliximab (INF)). In addition to the anti-TNF cohort, a parallel cohort of patients with active rheumatoid arthritis (RA) receiving non-biological disease-modifying antirheumatic drugs (nb-DMARD) has been recruited (guide disease activity score in 28 joints (DAS28) >4.2).

\section{Data collection}

Follow-up information is collected from medical records every 6 months for the first 3 years and annually thereafter. Data at follow-up include any changes to antirheumatic treatment, reasons for changes, and the onset of any adverse event including pregnancies. In addition, for the first 3 years of the study, patients are asked directly if they have received new treatments and about new referrals to (hospital) doctors. Data on pregnancies and pregnancy outcomes are also extracted from these patient reports.

All reports of pregnancies are followed up with an additional questionnaire which includes information on exposure to biological agents at the time of conception, details of pregnancy outcome including live births, spontaneous abortions and terminations. Details of pregnancy complications are collected as well as any details of congenital malformations. For the purpose of this analysis, pregnancies were divided into three groups: group I (exposure to anti-TNF at conception); group II (past exposure to anti-TNF); group III (never exposed to anti-TNF). Given the known risk of adverse pregnancy outcomes associated with the DMARDs methotrexate (MTX) and leflunomide (LEF), group I was further categorised into (a) those exposed to MTX and/ or LEF at conception and (b) those not 
exposed to MTX and/or LEF at conception. Women could have been included more than once in the analysis if more than one pregnancy had been recorded during the follow-up time and each pregnancy was allocated to the appropriate exposure group. For descriptive data, the denominator represents the number of pregnancies per group, and, therefore, the sum of the percentages presented within each group can be more than $100 \%$.

\section{RESULTS}

Study population

A total of 130 pregnancies in 118 women ever exposed to antiTNF agents and 10 pregnancies in 10 women never exposed to
anti-TNF agents were reported. At registration, baseline DAS28 and Health Assessment Questionnaire (HAQ) scores were significantly higher in the anti-TNF therapy groups than in the nbDMARD group (table 1). For both baseline DAS28 and HAO score, a significant difference was observed between groups Ia and $\mathrm{Ib}$. Patients in group Ia also had a higher HAQ score compared with patients in group II.

\section{Pregnancy outcomes}

Eighty-eight live births in a total of 130 pregnancies (including three pregnancies with twin gestation) in patients exposed to anti-TNF therapy were reported: $42 / 71$ (59\%) in group I and

Table 1 Overview of pregnancy outcomes in the BSRBR

\begin{tabular}{|c|c|c|c|c|}
\hline & $\begin{array}{l}\text { Group la: } \\
\text { anti-TNF therapy+ } \\
\text { MTX or LEF at time } \\
\text { of conception }\end{array}$ & $\begin{array}{l}\text { Group Ib: } \\
\text { anti-TNF therapy } \\
\text { but no MTX or LEF at } \\
\text { time of conception }\end{array}$ & $\begin{array}{l}\text { Group II: } \\
\text { anti-TNF therapy } \\
\text { prior to conception }\end{array}$ & $\begin{array}{l}\text { Group III: } \\
\text { never exposed to } \\
\text { anti-TNF therapy }\end{array}$ \\
\hline Number of women with pregnancy & 20 & 44 & 54 & 10 \\
\hline Single births & 21 & 49 & 58 & 9 \\
\hline Twins & 0 & 1 & 1 & 1 \\
\hline Age, mean (SD) & $29.7(8.1)$ & $34.4(5.2)$ & $32.6(4.9)$ & $32.5(5.2)$ \\
\hline Baseline $\mathrm{HAO}$ score, $\mathrm{n} / \mathrm{N}^{\dagger}$ & $20 / 20$ & $41 / 44$ & $49 / 54$ & $8 / 10$ \\
\hline Mean (SD) & $2.2(0.4)$ & $1.9(0.5)$ & $1.6(0.6)$ & $1.0(0.4)$ \\
\hline \multicolumn{5}{|l|}{ Diagnosis } \\
\hline RA & 16 & 36 & 46 & 10 \\
\hline PsA & 1 & 2 & 1 & 0 \\
\hline JIA & 2 & 4 & 4 & 0 \\
\hline Infliximab & 5 & 4 & - & - \\
\hline Etanercept & 12 & 36 & - & - \\
\hline Adalimumab & 4 & 10 & - & - \\
\hline \multicolumn{5}{|l|}{ Anti-TNF therapy prior to conception } \\
\hline Infliximab (INF) & 4 & 2 & 15 & 0 \\
\hline Etanercept (ETA) & 1 & 1 & 23 & 0 \\
\hline Adalimumab (ADA) & 1 & 0 & 11 & 0 \\
\hline$>1$ anti-TNF agent & 1 & 2 & 10 & 0 \\
\hline \multicolumn{5}{|c|}{ Conventional DMARD use at conception } \\
\hline Methotrexate (MTX) & 13 & 0 & 0 & 0 \\
\hline Leflunomide (LEF) & 3 & 0 & 0 & 0 \\
\hline Sulfasalazine (SSZ) & 0 & 1 & 4 & 3 \\
\hline Termination & $4(19 \%)$ & $4(8 \%)^{\pi}$ & $2(3 \%)$ & 0 \\
\hline Spontaneous abortion & $7(33 \%)$ & $12(24 \%)$ & $10(17 \%)$ & $1(10 \%)$ \\
\hline Neonatal death & 0 & $1(2 \%)$ & 0 & 0 \\
\hline Intrauterine death & 0 & $2(4 \%)^{\ddagger}$ & $2(3 \%)^{\ddagger}$ & 0 \\
\hline Premature delivery ( $\leq 36$ weeks) & 3 & $8^{\ddagger}$ & 8 & 2 \\
\hline
\end{tabular}

*DAS28 score significantly higher in the anti-TNF groups compared with the nb-DMARD group. DAS28 significantly higher in group la compared with group II ( $\mathrm{p}=0.0213$, unpaired $\mathrm{t}$ test). ** Since the number of outcomes is divided by the number of pregnancies, the total sum of percentages can be more than $100 \%$

${ }^{\dagger} \mathrm{HAO}$ score significantly higher in the anti-TNF groups compared with the nb-DMARD group. HAQ score significantly higher in group la compared with group Ib ( $\mathrm{p}=0.0353$ ) and significantly higher in group la compared with group II $(\mathrm{p}<001)$.

${ }^{\ddagger}$ Including one of twins.

§Including twins.

"One because of Down's syndrome.

DAS28, disease activity score in 28 joints; $\mathrm{HAO}$, Health Assessment Questionnaire; $\mathrm{n} / \mathrm{N}$, number of patients with available data/number of women with pregnancy; RA = rheumatoid arthritis; PsA = psoriatic arthritis ; JIA = juvenile idiopathic arthritis ; $\mathrm{AS}=$ ankylosing spondylitis; SLE = Systemic Lupus Erythematosus. 
46/59 (78\%) in group II; 10/10 (100\%) live births were reported in the nb-DMARD group. In two of the three twin pregnancies (one in group I and one in group II), one of the fetuses died in utero and one was delivered at/near term (table 1). The rate of spontaneous abortion was highest among patients exposed to anti-TNF at the time of conception (group I): overall 19/71 (27\%); with MTX/LEF 7/21 (33\%); without MTX/LEF $12 / 50$ (24\%). This compared with $10 / 59$ spontaneous abortions $(17 \%$ of pregnancies) in those with prior exposure to anti-TNF agents (group II) and $1 / 10$ spontaneous abortion (10\% of pregnancies) of those never exposed to anti-TNF (group III). In total there were 10 terminations ( 4 in group Ia, 4 in group Ib and 2 in group II). Of the 42 live births or 22 spontaneous abortions/intrauterine or neonatal deaths in women who were receiving anti-TNF therapy at the time of conception (group I), 33/42 (79\%) and $18 / 22(82 \%)$, respectively, discontinued anti-TNF therapy during the first trimester, $5 / 42(12 \%)$ and $0(0 \%)$ during the second trimester and $4 / 42(9 \%)$ and $1 / 22(5 \%)$ received anti-TNF therapy throughout their pregnancy. No data on anti-TNF exposure during pregnancy was available for 0 and 3 pregnancies, respectively. In those patients with prior exposure to anti-TNF, the length of time since the last dose of anti-TNF therapy did not differ between those with a live birth and those who miscarried (median 7.1 months (IOR 4.4-13.2) vs median 5.7 months (IOR $0.1-10.4) ; p=0.275)$.

\section{Pregnancy complications}

Of 88 live births in the anti-TNF groups, 19 babies (22\%) were born prematurely $(11 / 42(26 \%)$ in group I and $8 / 46(17 \%)$ in group II) compared with $2 / 10(20 \%)$ in the nb-DMARD group. One full-term baby had a low birth weight. Four fetuses died in utero (two in group Ib and two in group II), including two single fetuses in two twin pregnancies. One neonatal death $27 \mathrm{~h}$ after delivery was reported in a patient who received ETA during the first trimester. The cause of death was perinatal hypoxia. There were four reports of congenital malformations, two in group $\mathrm{Ib}$ (congenital dislocation of the hip and pyloric stenosis) and two in group II (winking jaw syndrome and strawberry birth mark).

\section{DISCUSSION}

Our study presents the results of the largest detailed prospective collection of pregnancy outcomes in women with arthritis-related diseases exposed to anti-TNF therapy. In our study population a potential signal of an increased spontaneous abortion rate was observed in women exposed to anti-TNF therapies at conception, although this was most evident in those also receiving MTX or LEF (33\%). Two general population-based studies found a clinical spontaneous abortion rate of around $12 \%,{ }^{4} 5$ although the actual rate may be higher ( 25\%) if clinically undetectable pregnancies are also included. ${ }^{6}$

To put these results into context with the evidence currently available, it is important to compare our findings with those published in previous reports (see table in online supplement). ${ }^{7-15}$ of the known outcomes in pregnancies in cohort studies including any patients exposed to anti-TNF agents before or during pregnancy, the spontaneous abortion rate ranged from $0 \%$ to $14 \% .{ }^{15}$ In one study, including data from a survey of US rheumatologists $^{8}$, one spontaneous abortion occurred in a patient using ETA in combination with MTX at the time of conception. Across all studies, 31 terminations were reported. . $^{7-9} 121415$ One pregnancy was terminated because of concomitant MTX use at the time of conception. ${ }^{7}$ Eleven babies were born with complications or neonatal illnesses. 9101415 Data from OTIS, published only in abstract form, show that the spontaneous abortion rate was $7.4 \%$ in patients exposed to $\mathrm{ADA}$ during the first trimester and $6.1 \%$ in the RA comparison group. These percentages are lower than we found in our study population, but no information regarding DMARD intake at conception and general disease activity was provided for the OTIS population. ${ }^{16}$

Both MTX and LEF are ' $\mathrm{X}$ ' category drugs. There is a recognised association between high-dose MTX and spontaneous abortion. A recent systematic review found that $23 \%$ of pregnancies exposed to MTX in the first trimester resulted in spontaneous abortion and $5 \%$ of pregnancies resulted in reported minor neonatal malformations. ${ }^{17}$ In our study, 20 patients became pregnant while receiving ' $X$ ' class drugs. It is not known whether they were informed about the detrimental effects of these drugs, although we do know that a few patients became pregnant while using oral contraceptives which suggests that these were unplanned pregnancies. However, it cannot be concluded that the concurrent use of MTX and/or LEF is the only explanation for this increased rate of spontaneous abortion, as the rate of spontaneous abortion in women exposed to anti-TNF therapy at conception without these drugs was also higher than in those previously or never exposed to anti-TNF agents. There are data to suggest that women with severe RA may have an unfavourable pregnancy outcome, and those patients unable to discontinue anti-TNF therapies may be those with the most severe disease. ${ }^{18} 19 \mathrm{We}$ did not collect data on disease activity at time of conception. It is thus important to weigh the impact of disease severity on pregnancy outcome and the results found in our study with regard to the somewhat increased risk of spontaneous abortions in patients receiving anti-TNF agents at conception. Despite the exposure of anti-TNF therapy at conception, few patients opted for termination. Compared with the termination rate of $12.9 \%$ in women aged $30-34$ years in the general population of England and Wales (ie, the percentage of pregnancies resulting in one or more live births or a stillbirth or legal abortion that were terminated by abortion), ${ }^{20}$ the termination rate was higher in those exposed to anti-TNF therapy plus MTX or LEF at conception (19\%) but lower in those exposed to anti-TNF agents alone (8\%).

Data on drug safety during pregnancy are largely restricted to the cumulative experience of patients and physicians and often limited to case reports. One of the biggest challenges in obtaining safety data is ensuring that outcomes in all exposed patients are recorded, not just those with particularly good or bad outcomes. The BSRBR, through the systematic follow-up of patients, has captured all pregnancy outcomes as they have occurred since the study started in 2001, including information on terminations, spontaneous abortions as well as pregnancy complications. This may also in part explain the higher rate of spontaneous abortion observed in this study compared with previous reports. We were also able to compare the pregnancy outcomes of patients exposed to anti-TNF therapy before or at conception with a control group of patients with RA who were never exposed to anti-TNF therapy. However, the number of recorded pregnancies in this control group was small.

\section{CONCLUSION}

The results of this current study, one of the largest detailed prospective studies to date, suggest that treatment with anti-TNF therapy at the time of conception may be associated with an increased risk of spontaneous abortion, but the role of disease 
severity and other antirheumatic treatment cannot be excluded. Although the collected results to date have been promising with few reports of congenital malformations, no firm conclusions can be drawn about the safety of anti-TNF therapy during pregnancy and, without further evidence, guidelines which suggest these drugs should be avoided at the time of conception must remain.

Acknowledgements The authors acknowledge the enthusiastic collaboration of all consultant rheumatologists and their specialist nurses in the UK in providing the data. In addition, we acknowledge the support from Dr lan Griffiths (Past) and Professor David Isenberg (Current), Chairs of the BSRBR Management Committee, Professor Gabriel Panayi, Professor David G I Scott, Dr Andrew Bamji and Dr Deborah Bax, Presidents of the BSR during the period of data collection, for their active role in enabling the Register to undertake its tasks and Samantha Peters (CEO of the BSR), Mervyn Hogg, Nia Taylor and members of the BSRBR Scientific Steering Committee. We also acknowledge the seminal role of the BSR Clinical Affairs Committee for establishing national biologic guidelines and recommendations for such a Register. Finally we would like to acknowledge the substantial contribution of Andy Tracey, Katie McGrother and Dr Mark Lunt to database design and manipulation and Professor Alan Silman in his prior role as principal investigator of the BSRBR.

Funding Funding for this project was provided by the British Society for Rheumatology (BSR). The BSR commissioned the Biologics Register (BSRBR) as a UK-wide national project to investigate the safety of biologic agents in routine medical practice. DPMS and $\mathrm{KH}$ are principal investigators on the BSRBR. BSR receives restricted income from UK pharmaceutical companies, presently Abbott Laboratories, Biovitrum, Shering Plough, Wyeth Pharmaceuticals and Roche. This income finances a wholly separate contract between the BSR and the University of Manchester. The principal investigators and their team have full academic freedom and are able to work independently of the pharmaceutical industry. All decisions regarding analyses, interpretation and publication are made autonomously of any industrial contribution.

Competing interests Members of the Manchester team, BSR trustees, committee members and staff complete an annual declaration in relation to conflicts of interest. The authors declare no other conflict of interest.

Patient consent Obtained.

Ethics approval The study received ethical approval from the UK North West Research Ethics Committee (MREC 00/8/53).

Provenance and peer review Not commissioned; externally peer reviewed.

\section{REFERENCES}

1. Skomsvoll JF, Wallenius M, Koksvik HS, et al. Drug insight: anti-tumor necrosis factor therapy for inflammatory arthropathies during reproduction, pregnancy and lactation. Nat Clin Pract Rheumatol 2007;3:156-64.

2. Carter JD, Ladhani A, Ricca LR, et al. A safety assessment of tumor necrosis factor antagonists during pregnancy: a review of the Food and Drug Administration database. J Rheumatol 2009;36:635-41.
3. Hyrich KL, Symmons DP, Watson KD, et al. Pregnancy outcome in women who were exposed to anti-tumor necrosis factor agents: results from a national population register. Arthritis Rheum 2006;54:2701-2.

4. Everett C. Incidence and outcome of bleeding before the $20^{\text {th }}$ week of pregnancy: prospective study from general practice. BMJ 1997;315:32-4.

5. Blohm F, Fridén B, Milsom I. A prospective longitudinal population-based study of clinical miscarriage in an urban Swedish population. BJOG 2008;115:176-82; discussion 183

6. Wilcox AJ, Weinberg CR, O'Connor JF, et al. Incidence of early loss of pregnancy. N Engl J Med 1988;319:189-94.

7. Berthelot JM, De Bandt M, Goupille P, et al. Exposition to anti-TNF drugs during pregnancy: outcome of 15 cases and review of the literature. Joint Bone Spine 2009;76:28-34.

8. Chakravarty EF, Sanchez-Yamamoto D, Bush TM. The use of disease modifying antirheumatic drugs in women with rheumatoid arthritis of childbearing age: a survey of practice patterns and pregnancy outcomes. J Rheumatol 2003;30:241-6.

9. Cush JJ. Biological drug use: US perspectives on indications and monitoring Ann Rheum Dis 2005;64:iv18-23.

10. Mahadevan U, Kane S, Sandborn WJ, et al. Intentional infliximab use during pregnancy for induction or maintenance of remission in Crohn's disease. Aliment Pharmacol Ther 2005;21:733-8.

11. Kane S, Ford J, Cohen R, et al. Absence of infliximab in infants and breast milk from nursing mothers receiving therapy for Crohn's disease before and after delivery. J Clin Gastroenterol 2009;43:613-6.

12. Ostensen $\mathbf{M}$, Lockshin $\mathrm{M}$, Doria $\mathrm{A}$, et al. Update on safety during pregnancy of biological agents and some immunosuppressive anti-rheumatic drugs. Rheumatology (Oxford) 2008;47:iii28-31.

13. Rosner I, Haddad A, Boulman N, et al. Pregnancy in rheumatology patients exposed to anti-tumour necrosis factor (TNF)-alpha therapy. Rheumatology (Oxford) 2007; 46:1508; author reply 1508-9.

14. Roux CH, Brocq 0, Breuil V, et al. Pregnancy in rheumatology patients exposed to anti-tumour necrosis factor (TNF)-alpha therapy. Rheumatology (Oxford) 2007:46:695-8.

15. Katz JA, Antoni $\mathrm{C}$, Keenan GF, et al. Outcome of pregnancy in women receiving infliximab for the treatment of Crohn's disease and rheumatoid arthritis. Am J Gastroentero/ 2004;99:2385-92.

16. Johnson DL, Jones KL, Chambers C. Pregnancy outcomes in women exposed to Adalimumab: OTIS autoimmune diseases in pregnancy project. Ann Rheum Dis 2008:58:S682

17. Martínez Lopez JA, Loza E, Carmona L. Systematic review on the safety of methotrexate in rheumatoid arthritis regarding the reproductive system (fertility, pregnancy, and breastfeeding). Clin Exp Rheumatol 2009;27:678-84

18. de Man YA, Hazes JM, van der Heide $\mathrm{H}$, et al. Association of higher rheumatoid arthritis disease activity during pregnancy with lower birth weight: results of a national prospective study. Arthritis Rheum 2009;60:3196-206.

19. Skomsvoll JF, Ostensen M, Irgens LM, et al. Pregnancy complications and delivery practice in women with connective tissue disease and inflammatory rheumatic disease in Norway. Acta Obstet Gynecol Scand 2000;79:490-5.

20. National Office for Statistics. Conceptions. 2010: http://www.statistics.gov.uk. (Data obtained 2009). 